\title{
Ein höheres Inflationsziel für die EZB?
}

Die Europäische Zentralbank (EZB) stellt derzeit ihre geldpolitische Strategie und dabei insbesondere die Konkretisierung inres Inflationsziels zur Debatte. Bisher definiert

(c) Der/die Autor(en) 2020. Open Access: Dieser Artikel wird unter der Creative Commons Namensnennung 4.0 International Lizenz (https:// creativecommons.org/licenses/by/4.0/deed.de) veröffentlicht.

Open Access wird durch die ZBW - Leibniz-Informationszentrum Wirtschaft gefördert. die EZB die Umsetzung des vertraglich vorgegebenen Ziels der Preisniveaustabilität als eine Inflationsrate auf mittlere Sicht von unter, aber nahe an $2 \%$ pro Jahr. Diskutiert wird nun, dieses Ziel auf $2 \%$ leicht anzuheben und seine Erreichung im Durchschnitt mehrerer Jahre anzustreben (Lagarde, 2020). Beide Elemente sind durchaus überraschend, da die realisierte Inflationsrate in den vergangenen Jahren eher tiefer lag und da ein mehrjähriger Durchschnitt den Spielraum der EZB für eine expansivere 
Prof. Dr. Bernhard Herz ist Inhaber des Lehrstuhls für VWL, insbesondere Geld und Internationale Wirtschaft, an der Universität Bayreuth.

Prof. Dr. Lukas Menkhoff lehrt VWL an der Humboldt-Universität zu Berlin und leitet die Abteilung Weltwirtschaft am DIW Berlin.

Geldpolitik nochmals erweitert. Warum läuft die Diskussion dennoch in diese Richtung und was sind die Pro- und Contra-Argumente?

\section{Rückblick auf Strategie-Entwicklung}

Die Entwicklung der Strategie der EZB versteht man am besten mit einem Blick auf ihre Entstehungsgeschichte. Als der Plan einer neu zu gründenden EZB in den 1990er Jahren konkretisiert wurde, war das Rollenmodell einer erfolgreichen Zentralbank in Europa sicherlich die Deutsche Bundesbank. Diese hatte eine beeindruckende Geschichte an Preisniveaustabilität in einem inflationären internationalen Umfeld vorzuweisen. Seit den 1970er Jahren hatte sie als erste Zentralbank Geldmengenziele eingeführt und diese aus Projektionen abgeleitet. Teil dieser Begründung ihrer Politik war die Annahme einer damals „unvermeidbaren“ Preisniveausteigerung, die seit 1985 pro Jahr $2 \%$ betrug.

Diese $2 \%$ waren in erster Linie ein pragmatisch gewählter Wert, denn in den 1970er Jahren und bis in die frühen 1980er Jahre waren die Inflationsraten auch in Deutschland recht hoch, bis zu etwa $6 \%$ bis $7 \%$ pro Jahr. Vor diesem Hintergrund waren $2 \%$ ein sehr moderater Wert für eine akzeptierte Inflationsrate und sollten einen Anker für die Rückführung und Ausbildung stabiler Inflationserwartungen bilden. Tatsächlich hat die Bundesbank, insbesondere ihr langjähriger Chefökonom und zeitweiliger Präsident Helmut Schlesinger (1976), auch Inflationsraten deutlich unter $2 \%$ nicht als abwegig angesehen.

Bei der Konzeption der EZB-Strategie lag es also nahe, sich am Modell der Bundesbank zu orientieren, zumal mit Ottmar Issing, dem früheren Chef-Ökonom der Bundesbank, ein intimer Kenner des Geldmengenkonzepts auf die Stelle des ersten EZB-Chefökonomen gewechselt war. Der ursprüngliche Ansatz war als Zwei-Säulen-Strategie formuliert, wobei die erste Säule unmittelbar an der Tradition der Bundesbank mit der Ableitung eines Geld-

\section{Abbildung 1}

Inflation im Euroraum und in den USA sowie Ölpreis Änderungsrate des Harmonisierten Verbraucherpreisindex HVPI, in \% (linke Skala), Ölpreis Brent $1999=100$ (rechte Skala)

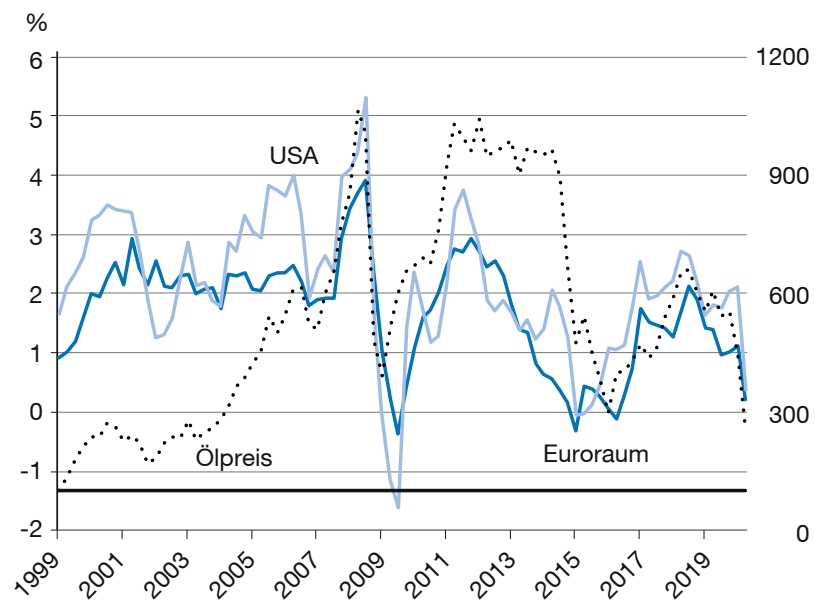

Quellen: Eurostat (2020); OECD (2020); U.S. EIA (2020); eigene Darstellung.

mengenziels anschloss, während die zweite Säule eine breite Analyse des Preisniveauumfelds formulierte. Die Zielgröße war ein Preisniveauanstieg von unter $2 \%$ pro Jahr, gemessen am neu eingerichteten Harmonisierten Verbraucherpreisindex (HVPI).

Als Ergebnis der Strategieüberprüfung nach den ersten Praxisjahren der EZB kam es zu zwei Änderungen. Zum einen wurden die beiden Säulen nun in eine neue Rangordnung gebracht, indem das „alte“ Geldmengenziel weicher formuliert und als zweitrangig betrachtet wurde. Dagegen wurde die Analyse des Preisniveauumfelds in den Vordergrund gestellt. Die zweite Änderung war eine leichte Präzisierung des Preisniveauziels auf die heutige Formulierung. Im Grunde hatte die EZB damit den Wandel von einer Geldmengenstrategie (wie sie die Bundesbank formal praktiziert hatte) zu einem Inflationsziel vollzogen.

Seit dem Start als geldpolitisch verantwortliche Institution zum 1.1.1999 hat die EZB ihr Ziel der Preisniveaustabilität im Großen und Ganzen gut erreicht. Die Inflationsraten haben sich im Euroraum fast immer in einer Spanne von etwa $1 \%$ bis $3 \%$ bewegt, mit leicht sinkender Tendenz (vgl. Abbildung 1). Insbesondere die beiden großen Wirtschaftskrisen 2008/2009 und 2020, die Staatschuldenkrise Mitte der vergangenen Dekade und Ölpreisschocks sind gut zu erkennen. Ein internationaler Vergleich macht gleichzeitig deutlich, wie sehr sich die Inflationsraten seit den 1970er Jahren verändert haben. Auch die Notenbanken anderer Industrie- und Schwel- 
Abbildung 2

Inflationsraten in zahlreichen Mitgliedsländern der Eurozone 2019

Änderungsrate des Harmonisierten Verbraucherpreisindex HVPI, in \%

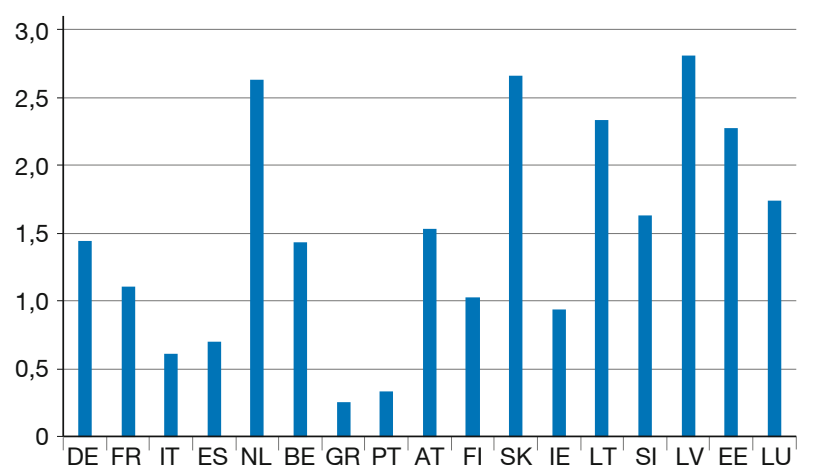

Anmerkung: Länderkürzel nach ISO-3166 Alpha-2. Reihenfolge der Länder nach Bruttoinlandsprodukt geordnet.

Quelle: OECD (2020); eigene Darstellung.

lenländer, wie die US-amerikanische Zentralbank Federal Reserve (Fed), „erreichen“ ein vergleichbar hohes Maß an Preisniveaustabilität wie die EZB.

\section{Deflationsgefahr als aktuelles Problem?}

Aufgrund der zuletzt oft niedrigen Inflationsraten wird diskutiert, ob die EZB ihr Inflationsziel verfehlen könnte und stattdessen dem Euroraum eine Deflation droht. Dies ist eine der Begründungen für die überaus expansive Geldpolitik der vergangenen Jahre. Tatsächlich sind die durchschnittlichen Inflationsraten aber in der Spanne von 1\% bis $2 \%$ geblieben, und nur große Krisen und der Rückgang der Rohstoffpreise drückten die Inflationsraten unter diese Spanne. Allerdings mag dies zum Teil der expansiven Geldpolitik zu verdanken sein. Darüber hinaus wird auch angeführt, dass die strukturellen Veränderungen im Rahmen der Globalisierung mit der Ausweitung des Arbeitskräftepotentials sowie die nachlassende Dynamik alternder Gesellschaften dazu geführt haben, dass überall auf der Welt die Inflationsraten niedrig geblieben sind (Summers, 2014; vgl. auch Abbildung 1).

Immerhin, wenn man Deflationsgefahr als zentrales Problem sieht, dann ist eine Erhöhung des Inflationsziels eine durchaus zielkonforme Maßnahme. Die Hoffnung besteht, dass unter einer glaubwürdigen Forward Guidance die Inflationserwartungen erhöht werden können, ohne dass die Geldpolitik kurzfristig aktiv werden muss (in den USA hat das erhöhte Inflationsziel bisher keinen Niederschlag in den Inflationserwartungen der Haushalte gefunden, Coibion et al., 2020). Mittelfristig allerdings ist die EZB gefordert, ihr Ziel dann auch zu realisieren.

\section{Abbildung 3}

Inflation im Euroraum: Tatsächlich und erwartet

2- und 5-Jahres-Prognose, Änderungsrate des Harmonisierten Verbraucherpreisindex $\mathrm{HVPI}$, in \%

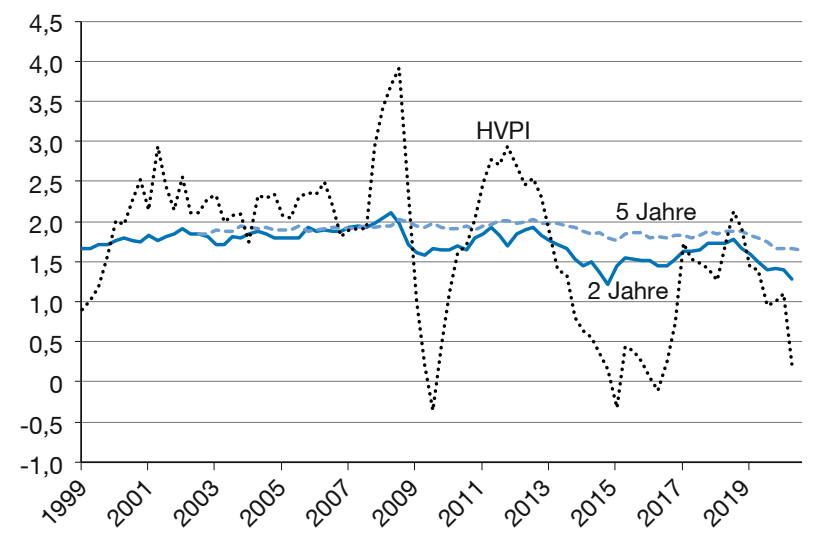

Quellen: EZB (2020); Eurostat (2020); eigene Darstellung.

Ein möglicherweise größeres Problem als die durchschnittliche Inflationsrate stellt die Heterogenität im Euroraum dar. Die Inflationsraten in den teilnehmenden Ländern können sehr unterschiedlich hoch sein. Zwar nahmen im Laufe der Zeit nicht nur die Inflationsraten ab, sondern auch die Unterschiede innerhalb des Euroraums. Dennoch bleiben teilweise deutliche Unterschiede wie beispielsweise 2019 (vgl. Abbildung 2). Es ist daher nicht auszuschließen, dass einzelne, vor allem kleinere Volkswirtschaften Phasen mit negativen Inflationsraten bei gleichzeitig positiver durchschnittlicher Inflation im Euroraum durchlaufen.

Sind nun vorübergehend negative Inflationsraten eine größere Gefahr als vorübergehend positive? Allgemein betonen Notenbanken die Gefahren, dass aus vorübergehend negativen Inflationsraten die Erwartung dauerhaft sinkender Preise entsteht, mit entsprechenden negativen Folgen für die Güternachfrage und damit die wirtschaftliche Entwicklung (Europäische Zentralbank 2011). Die zentrale Größe sind demnach die Inflationserwartungen. Im Euroraum sind diese aber recht stabil. Private Haushalte, Unternehmen und Expert*innen scheinen zu verstehen, dass rückläufige, auch negative Inflationsraten exogenen Schocks geschuldet sind, und erwarten, dass die EZB weiterhin an ihrem ursprünglichen Inflationsziel festhält. Insbesondere die Inflationserwartungen für den 5-Jahreszeitraum sind sehr persistent im Vergleich zu den aktuellen Inflationsraten. Damit sind sie fest entsprechend des EZB-Inflationsziels verankert und halten groBen Abstand zur Nullgrenze (vgl. Abbildung 3). Allerdings reagieren gerade die kürzerfristigen Erwartungen leicht auf die jeweils aktuelle Inflationsentwicklung (Möhrle und 
Wollmershäuser, 2020), sodass die Divergenz zu einem erhöhten Inflationsziel steigt.

\section{Folgen unkonventioneller Geldpolitik}

Die EZB hat in ihren Verlautbarungen die Gefahr möglicher Deflation immer betont, um ihre expansive Geldpolitik zu begründen. $\mathrm{Da}$ in der Folge der weltweiten Wirtschaftsund Finanzkrise 2008/2009 und der folgenden Staatschulden- und Bankenkrise im Euroraum die kurzfristigen Zinssätze bereits nahe an der Nullgrenze angekommen waren, folgte die EZB dem Beispiel der Fed und nutzt seitdem neuere Instrumente der sogenannten „unkonventionellen“" Geldpolitik. Im Wesentlichen geht es dabei um den Ankauf von Wertpapieren, um die Zentralbankgeldmenge zu erhöhen und die langfristigen Zinssätze niedrig zu halten. Ein - nicht unerwünschter - Nebenaspekt dieser Politik ist es, eine erhebliche Nachfrage vor allem auch nach Staatsanleihen von Mitgliedsländern zu generieren.

In den meisten empirischen Studien zu diesem Thema führt die unkonventionelle Geldpolitik verschiedener Zentralbanken zu sinkenden Zinssätzen und in der Regel $z u$ einer expandierenden Realwirtschaft (Bernoth et al., 2015). Die Debatte kreist also weniger um die Tatsache einer Wirkung, sondern um das Nutzen-Kosten-Verhältnis. Die EZB argumentiert, dass die Wachstumswirkung ohne Inflation bei Gütern und Dienstleistungen erzielt werden kann, also ihr Hauptziel nicht gefährdet sei. Kritiker*innen dagegen weisen auf eine Inflationswirkung bei den Vermögenspreisen hin.

Es fällt schwer, letztere zu negieren, denn sie ergibt sich logisch aus der expansiven Geldpolitik. In gängigen $\mathrm{Be}-$ wertungsmodellen für Vermögenspreise geht der risikolose Zinssatz als Diskontierungsfaktor ein, und wenn dieser nahe bei null liegt, dann führt die Berücksichtigung langer Perioden zukünftiger Erträge eines Vermögensgegenstands folgerichtig zu fast beliebig hohen Bewertungen. Dies ist vereinbar mit der empirischen Illustration in Abbildung 4, in der für die Jahre nach der großen Wirtschaftskrise die Bilanzsumme der EZB (als Proxy für ihre unkonventionelle Geldpolitik), das nominale Bruttoinlandsprodukt des Euroraums und der Euro Stoxx 600 als Maß für die Entwicklung der Aktienmärkte im Euroraum einander gegenübergestellt werden (Deutsche Bundesbank, 2019, zu Wohnimmobilien).

\section{Erhöhtes, mehrjähriges Inflationsziel?}

Vor diesem Hintergrund ist nun erstaunlich, dass - in Anlehnung an die Fed - diskutiert wird, das Inflationsziel leicht zu erhöhen und seine Erreichung über Jahre zu strecken. Eigentlich könnte man erwarten, dass ein ver-
Abbildung 4

EZB-Bilanzsumme, Bruttoinlandsprodukt und Europäischer Aktienindex Euro Stoxx 600

$2011=100$

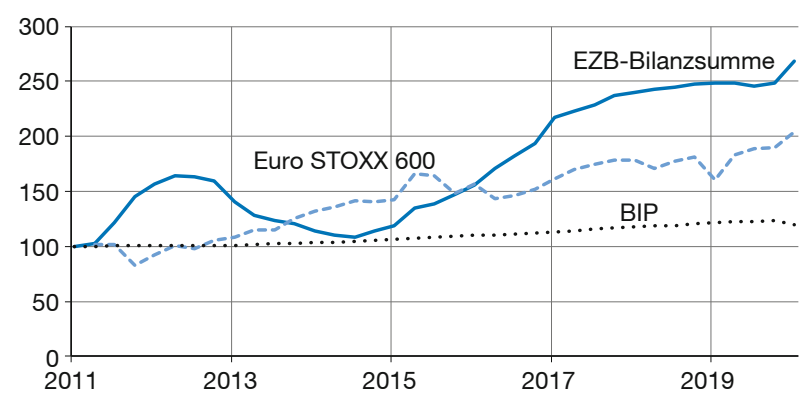

Quellen: EZB (2020); Eurostat (2020); Thomson Reuters Datastream (2020); eigene Darstellung.

gebliches Bemühen um Erreichung des Inflationsziels trotz extrem expansiver Politik - zum Nachdenken Anlass gibt, das Ziel möglicherweise nach unten anzupassen, denn offensichtlich fällt es der Geldpolitik schwer, höhere Inflationsraten umzusetzen. Es gibt gute Gründe, gerade aufgrund der Globalisierung, warum die Inflation so gering ist. Und falls tatsächlich hohes und billiges Arbeitskräftepotenzial die Inflation eindämmt, dann mündet diese nicht in Deflation, sondern beschreibt ein Gleichgewicht.

Die Formulierung eines mehrjährigen Inflationsziels hätte aber auch weitreichende Politikfolgen. Schon heute richtet die EZB inre Geldpolitik auf die mittlere Frist aus und trägt damit dem Umstand Rechnung, dass die Transmissionsverzögerungen nicht nur lang, sondern auch zeitlich und von der Größe her variabel sind (Europäische Zentralbank, 2011; Gischer et al., 2020). Sie versucht also nicht, einen deflationären Gütermarktschock sofort und vollständig zu neutralisieren, sondern toleriert Abweichungen und strebt mittelfristig wieder eine Inflationsrate von knapp unter $2 \%$ an. Unter einem Regime mehrjähriger Inflationsziele, wie von der Fed im Sommer angekündigt und von Präsidentin Lagarde auch für die EZB ins Spiel gebracht, würde die EZB aber auf einen (vorübergehenden) Rückgang der Inflationsrate unter den Zielwert mit einer Geldpolitik reagieren, welche die Inflationsrate zukünftig für den gleich langen Zeitraum entsprechend über den Zielwert hebt. Die Geldpolitik ist damit nicht mehr nur zukunftsgerichtet, sondern reagiert auch auf Entwicklungen der Vergangenheit. Der frühere EZB-Vizepräsident Christian Noyer hält dies für eine „extrem riskante" Strategie (Handelsblatt 2020). So müsste die EZB dann etwa nach einer zehnjährigen Unterschreitung des Inflationsziels um einen Prozentpunkt eine Dekade lang einen Wert von $3 \%$ erreichen, um den Durch- 
schnitt von $2 \%$ zu realisieren. Dies wäre offensichtlich mit enormen Kosten für die Wirtschaft verbunden. Es müsste dann auch mit einer volatileren Entwicklung der Inflationsrate gerechnet werden.

Die Überlegung eines erhöhten mehrjährigen Inflationsziels bedeutet demnach nichts anderes, als die bisherige Geldpolitik auf mittlere Sicht fortzusetzen. Stärker noch, die Inflationserwartungen und dann die Inflationsrate sollen sozusagen mit Gewalt auf die $2 \%$ gebracht werden. Man muss sich seiner Sache schon sehr sicher sein, um den Einsatz weiter zu erhöhen, also um eine vervielfachte Zentralbankbilanz (und Zentralbankgeldmenge) nochmals kräftig auszuweiten.

\section{Zusätzliches Risiko}

Die angestellten Überlegungen erhöhen nicht nur die Kosten der bisherigen Politik im Sinne einer verlängerten Phase inflationierter Vermögenspreise, sondern sie gefährden möglicherweise auch die Stabilität des Geldwesens. Historisch gesehen war es immer leicht gewesen, Geldentwertung zu erzeugen. Dabei ging es in der Vergangenheit typischerweise um eine inflationär finanzierte Staatsverschuldung. Jetzt hingegen sieht es so aus, als sollte einer drohenden Deflation entgegengewirkt werden. Doch so verschieden sind die ökonomischen Vorgänge nicht, dass man sicher sein kann, dass die gegenwärtige Politik nicht abgleitet. Nicht, dass die EZB dies bewusst so steuert, aber riskiert sie nicht ihre Glaubwürdigkeit?

Zum einen ist es aus geldpolitischer Sicht eine riskante Strategie, mit viel Geld zu versuchen die Inflation anzuheizen, wenn man merkt, dass dies kaum gelingt. Es ist keine neue Einsicht, dass Inflation nicht nur ein monetäres Phänomen ist und jedenfalls nicht eins zu eins mit der (Zentralbank-)Geldmenge korreliert. Aber wenn dies stimmt, dann nicht nur heute, sondern auch in Zukunft, wenn die Inflationsrate - endlich? - über die $2 \%$ hinausgeht. Wenn die monetäre Expansion zurückgeführt wird, begrenzt dies dann automatisch die Inflation? Falls die EZB an Vertrauen verliert, kann sie auch die Kontrolle über einen Restriktionsprozess verlieren, und die Anpassungskosten können deutlich steigen.

Zum anderen kann es passieren, dass die Politik der EZB die eines Tages anstehende Restriktion unmöglich macht. Zurzeit ist dies schwer vorstellbar, aber es mag Szenarien geben, in denen die EZB nicht mehr so autonom handeln kann, wie es heute selbstverständlich erscheint. Die Unabhängigkeit von Zentralbanken ist nur eine Periode in deren Geschichte. Dies gilt umso stärker, je mehr sich die EZB auf eine Strategie einlässt, die heute attraktiv erscheint, deren Kosten aber erst später auftreten mögen.
Die angedeuteten Risiken wären besser zu beherrschen, wenn Europa eine dynamische wirtschaftliche Zukunft bevorstünde, so wie es viele Länder nach 1945 erlebt haben. Dann könnte die EZB die monetäre Expansion stoppen, die Realwirtschaft würde den bildlich gesprochen großen monetären Mantel allmählich füllen, die Staaten wüchsen im Erfolgsfall aus ihren Schulden heraus, und die Situation würde sich ohne schmerzhafte Anpassungsprozesse bereinigen. Doch ist das realistisch? Hat Europa nicht grundlegende strukturelle Probleme, nicht zuletzt eine alternde Bevölkerung, die niedrige Wachstumsraten viel realistischer erscheinen lassen?

\section{Rollenverständnis der EZB}

Sicher ist die derzeitige Wirtschaftslage, durch die coronabedingte Pandemie ausgelöst, eine enorme Herausforderung für die Wirtschaftspolitik. Und sicher war die Geldpolitik in Europa aufgrund der vereinheitlichten Entscheidung und des Verzichts auf eine direkte parlamentarische Kontrolle schnell handlungsfähig. Im Zusammenspiel der Politikbereiche hat die Geldpolitik den anderen Politikträgerinnen Spielräume verschafft, ohne dass Inflation zu einem Problem geworden ist. Dies sind aber keine guten Gründe dafür, dass die Geldpolitik nicht nur ihren aktuell äußerst expansiven Kurs mit dem neuen Ankaufprogramm Pandemic Emergency Purchase Programme (PEPP) noch einmal verstärkt, sondern auch ihre geldpolitischen Ziele expansiver ausrichtet.

\section{Literatur}

Bernoth, K., P. König und C. Raab (2015), Large-Scale Asset Purchases by Central Banks II: Empirical Evidence, DIW Roundup, 61.

Coibion, O., Y. Gorodnichenko, E. S. Knotek II und R. Schoenle (2020), Average Inflation Targeting and Household Expectations, Federal Reserve Bank of Cleveland, Working Paper, Nr. 20-26.

Deutsche Bundesbank (2010), Preisniveausteuerung als geldpolitische Strategie, Monatsbericht Januar, 31-46.

Deutsche Bundesbank (2019), Finanzstabilitätsbericht.

Europäische Zentralbank (2011), Die Geldpolitik der EZB.

Gischer, H., B. Herz und L. Menkhoff (2020), Geld, Kredit und Banken. Eine Einführung, 4. Aufl., Springer.

Handelsblatt (2020), Lagarde öffnet die Tür für ein flexibleres Inflationsziel, Handelsblatt, 30. November.

Lagarde, C. (2020), The monetary policy strategy review: some preliminary considerations, speech at the "ECB and Its Watchers XXI" conference, 30. November.

Möhrle, S. und T. Wollmershäuser (2020), Über die drohende Entankerung der Inflationserwartungen in der Eurozone und die Handlungsspielräume der EZB, ifo Schnelldienst, 73(10), 30-32.

Schlesinger, H. (1976), Geldpolitik in der Phase des Wiederaufbaus (19501958), in Deutsche Bundesbank (Hrsg.), Währung und Wirtschaft in Deutschland 1876-1975.

Summers, L. H. (2014), U.S. Economic Prospects: Secular Stagnation, Hysteresis, and the Zero Lower Bound, Business Economics, 49(2) 65-73. 